\title{
Applications of distributed opto-electronic sensing technologies in geotechnical engineering monitoring
}

\author{
Maocai Zhao ${ }^{*}$, Sergey Kudryavtsev ${ }^{2}$, Semen Bugunov²., Vyacheslav Shemyakin ${ }^{2}$, \\ Yuliya Bugunova2 1 \\ ${ }^{1}$ Harbin Institute of Technology, Harbin, China \\ ${ }^{2}$ Far Eastern State Transport University, Khabarovsk, Russia
}

\begin{abstract}
This paper summarizes the application status of distributed optical fiber sensing technology in the main fields of geotechnical engineering monitoring in China and Far Eastern Russia, including pile foundation engineering, foundation pit engineering, slope engineering, tunnel and bridge engineering monitoring.
\end{abstract}

\section{Introduction}

The most significant advantage of distributed optical fiber sensing technology is that it breaks through the concept of traditional point sensing, and can measure the strain, temperature, damage and other information at any point along the optical fiber line, so as to realize continuous distributed monitoring of the measured object and capture the overall strain characteristics of the measured object.At present, distributed optical fiber monitoring technology in geotechnical engineering monitoring mainly includes the following five successful applications.

\section{Monitoring technique}

RP1000 series high spatial resolution distributed Brillouin optical fiber temperature and strain analyzer, using latest DPP-BOTDA technology, possess spatial resolution up to $2 \mathrm{~cm}$ (fig.1).

It is the only optical fiber sensing system so far in the world which can realize temperature and strain measurement with 2 centimeter high spatial resolution and 2 kilometers long range measurement at the same time. The integrated performance of this series is in the leading position among its competing products.

The schematic diagram of Brillouin optical time-domain analysis Sensing (fig.2). BOTDA employs two counter-propagating lasers, i.e., a pump pulse and a $\mathrm{CW}$ probe wave,

* Corresponding author: olgakudr56@mail.ru 
and the Brillouin gain spectra (BGS) of the sensing optical fiber can be obtained by scanning the frequency offset between the pump pulse and the probe wave in the vicinity of the Brillouin frequency shift (BFS). According to the linear relationship between Brillouin frequency shift and optical fiber strain and temperature, the distribution of strain and temperature along the sensing optical fiber can be obtained by measured Brillouin frequency shift.

The advantages of BOTDA technology are as follows: 1. Strong signal, i.e. high signalto-noise ratio, 2. Long sensing distance, 3. High spatial resolution, 4. High measurement accuracy.
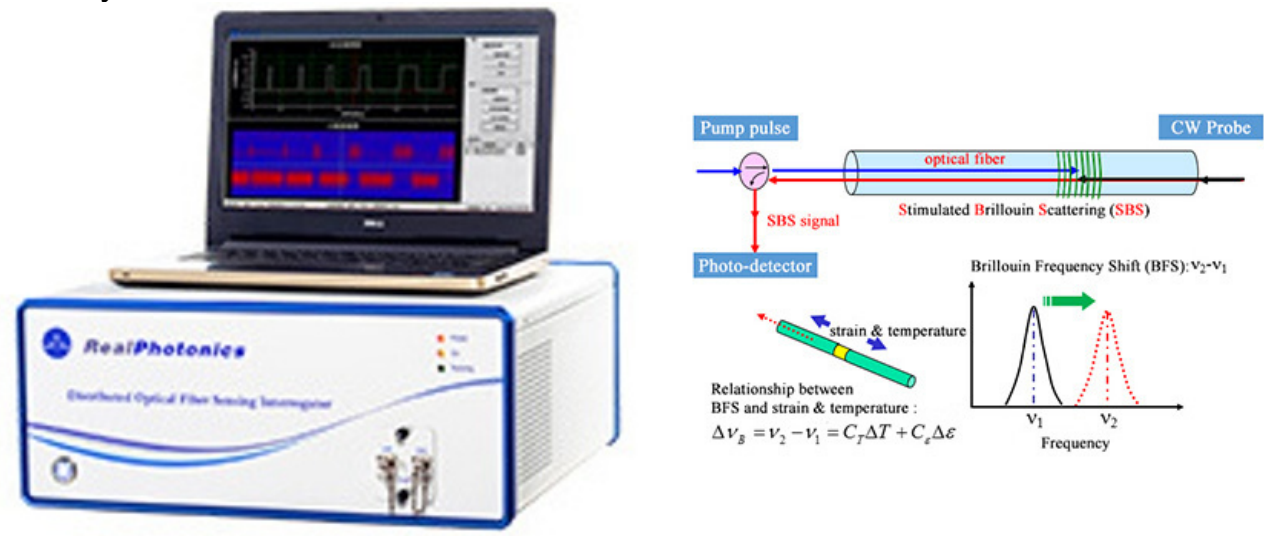

Fig. 1. Distributed optical fiber temperature and strain optical Time-Domain analyzer Brillouin

The schematic diagram of differential pulse pair technology (fig.2).
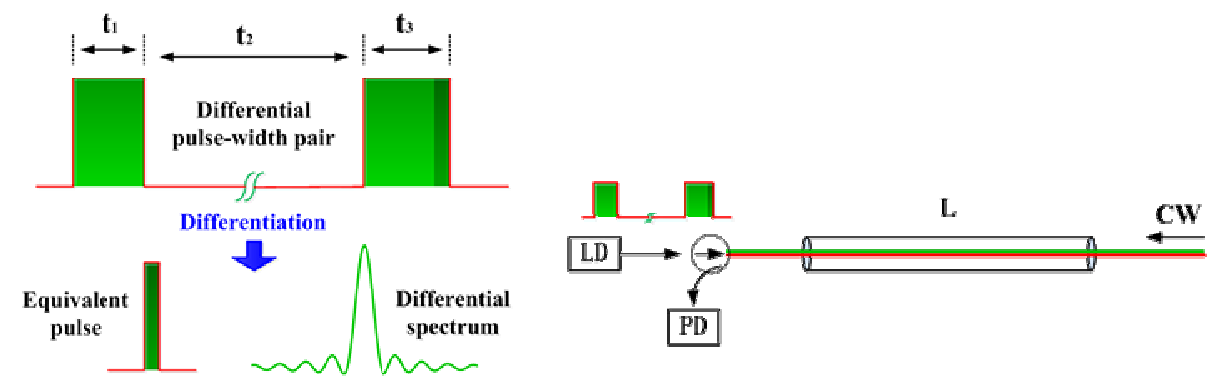

Differential pulse-width pair (DPP) BOTDA technique provides a high spatial resolution

Fig. 2. Differential pulse pair technology

The differential pulse pair technology is an innovative method which can significantly improve the spatial resolution of a BOTDA system. This technology has to be applied in two steps. Firstly, two time traces of the Brillouin gain signal over the same section of an sensing optical fiber are obtained by using two pulses with different pulse widths respectively. Secondly, differential Brillouin spectra are obtained by taking subtraction between the two traces of Brillouin signals and the corresponding temperature or strain can be obtained by demodulating the differential Brillouin spectra.

Concretely, two pump pulses with width of $t 1$ and $t 3$ successively input into the 
optical fiber with length of $\mathrm{L}$ in a time interval $\mathrm{t} 2(>2 \mathrm{~nL} / \mathrm{c})$. The injected pump pulse pair interact with the counter-propagating continuous wave laser and the Brillouin signals corresponding to the two pulse are received by the detector separately. Then subtraction was conducted to the two Brillouin signals to obtain the differential gain signal. Additionally, Brillouin gain spectra of each position along the optical fiber can be obtained by sweeping the frequency offset in the vicinity of the Brillouin frequency shift (BFS), and BFS of each sensing point is obtained by the curve fitting. Finally, the temperature or strain distribution of the optical fiber can be demodulated according to their relations to the frequency shift.

The advantages of differential pulse pair technology can be summarized in the following three aspects 1 . Strong Brillouin signal with high signal-to-noise ratio, 2. Brillouingain spectrum with narrow linewidth, 3. Long sensing distance with high spatial resolution and high precision sensing.

\section{Pile foundation engineering monitoring}

The main sensors used in pile foundation detection include reinforcement stress meter, slide micrometer, resistance strain gauge, etc. These are all point sensors, and the sensor elements are vulnerable to the impact of the surrounding environment and damage, low survival rate. The economics of distributed detection makes pile foundation detection develop from traditional point monitoring to distributed, high spatial resolution and high precision monitoring.

Application type steel strand single-mode tight set of optical cable, optical fiber in the outside through the many strands of metal reinforcement protection, greatly improve the surface strength, can well protective fiber, at the same time, can well solve the problem of coupled with reinforced concrete, pile body strain of the pile body is obtained under various load change law of axial force and side frictional resistance, and with the reinforcement stress meter contrasted the measured axial force, prove the validity of the distributed optical fiber monitoring technology.

The construction site monitoring of foundation pit engineering mainly includes envelop system and surrounding environment monitoring of foundation pit.Among them, precision level and theodolite are mainly used to monitor the settlement and horizontal displacement of the envelope top.Pile wall displacement of deep soil and the deflection of deep level usually USES the inclinometer measuring, but in the soil deformation is too large or the dip tube into the barrier, will cause the inclinometer probe can not reach the location on the dip tube, can not be implemented the normal monitoring, and need special personnel to carry on the field monitoring, difficult to realize automation and real-time monitoring;Pile wall, ring beam, pillar and support the structural internal force monitoring support by using vibrating string type reinforced stress meter, the resistance strain gauge and differential resistance type reinforcement stress meter, the soil layer anchor stress monitoring usually adopt anchor dynamometer and anchor reinforcement stress meter, the conventional testing instruments and sensors are widespread anti-interference and low durability and long-term stability of faults, it is difficult to meet the requirements of modern geotechnical engineering monitoring.At present, the techniques and methods applied in foundation pit engineering monitoring at home and abroad are developing from traditional point instrument monitoring to distributed, automatic, high-precision and remote monitoring.

The monitoring objects of foundation pit engineering mainly include diaphragm wall, row pile, soil anchor, ring beam, support, column, adjacent soil and underground pipeline. The sensor fiber used for distributed monitoring of foundation pit engineering is generally single mode communication fiber, which is easy to be damaged during construction. 
Therefore, it is necessary to take appropriate protection measures or special packaging to ensure that the sensor fiber will not be damaged during construction and later monitoring.

Foundation pit engineering based on the technology of distributed fiber optic monitoring remote distributed optical fiber monitoring system, using distributed sensing optical fiber in the containment system, dip tube, the soil pile, anchor and adjacent underground pipeline layout, installation and protection technology, as well as the temperature compensation method, and based on a deep foundation pit in high-rise building in Nanjing at the scene of the distributed optical fiber monitoring test and bolt drawing test, verify the feasibility of the technology applied in foundation pit engineering monitoring.

\section{Slope engineering monitoring}

The techniques and methods applied to slope engineering monitoring are developing from traditional point instrument detection to distributed, automatic, high-precision and remote monitoring. Conventional sensors, such as steel string type, resistance strain gauge type and inductance type, generally have the disadvantages of poor anti-interference, durability and long-term stability, which are difficult to meet the requirements of modern geotechnical engineering monitoring. In recent years, optical fiber sensors have the characteristics of anti - electromagnetic interference, water - proof, anti - corrosion and long durability. In particular, distributed optical fiber sensor is small in size and light in weight, which is easy to lay and install. There is no matching problem when it is implanted into the monitored object, which has little influence on the performance and mechanical parameters of the monitored object. Fiber itself is not only the sensor body but also the signal transmission medium.

Based on the arrangement of sensor fiber in slope reinforcement engineering, protection method of sensor fiber and temperature compensation method, the deformation deflection of anchor bolt stress and anti-slide pile is calculated, and the distributed fiber strain remote monitoring system for slope reinforcement engineering is constructed. The field experiment results show that the distributed optical fiber monitoring technology is feasible and effective for the strain and deformation monitoring of slope engineering.

\section{Tunnel engineering monitoring}

In Gulou tunnel health diagnosis, for example, the paper analyzes the distributed monitoring technology applied to the tunnel deformation monitoring and monitoring scheme, discusses the tunnel environmental factors such as temperature and vibration on the influence of the monitoring results, verify the sensitivity of monitoring system, and finally the result of the deformation monitoring of tunnel was analyzed, and the drum tower is given the health diagnosis of the tunnel.

Distributed monitoring technology is feasible and effective for strain monitoring of tunnel deformation, crack occurrence and development. The laying scheme of distributed fiber optic strain monitoring is successful and the laying technology is feasible. The monitoring results can be used as the basis of tunnel health diagnosis.

\section{Bridge engineering monitoring}

Due to the traditional bridge monitoring schemes are mainly point measurement, easy to cause residual, low reliability, if encryption setting cause workload multiplied, and each measurement must be preparations for a long time to live, also difficult to achieve long 
distance, large area monitoring, so need to find a more convenient and reliable monitoring technology to make up for these deficiencies.

The distributed fiber strain sensor monitoring technology was used to compare the measured results with the point digital strain sensor, the measured values of fiber Bragg grating and the modeling, analysis and calculation of finite element software.The results show that the local measurement values of distributed fiber optic sensing technology are consistent with the strain gauge and theoretical test, and the continuous distribution results of strain at the bottom of concrete t-beam during static load test can be measured.

Relying on the construction projects of several super large Bridges, the traditional monitoring method is compared with distributed optical fiber technology, and the results show that optical fiber technology has more accurate advantages and can more accurately reflect the internal strain of the structure, which proves the feasibility and superiority of its practical application. It is worth noting that the Amur bridge in Russia is using this technology for health monitoring.

Haicang bridge, which connects Xiaman Island and Haicang District and has been in service for 15 years, is the longest continuous floating steel box girder suspension bridge in Asia and the second longest in the world.The bridge serves as a key joint for state highways and urban transportation. Haicang Bridge represents the crowning achievement of the Chinese bridge construction technology advance in the 20th Century.

In 2015, our self-designed product "High Resolution Distributed Optical Fiber Temperature and Strain Analyzer" was used to conduct the structure health monitoring for Haicang Bridge during the load test.

The measured parameters included temperature and strain distributions and were obtained by the three sensing optical fibers installed on the steel box girders covering the whole length of $1180 \mathrm{~m}$ of the bridge. The load test were divided into four different working conditions by placing the load trucks at different locations and the corresponding strain distributions along the whole length of the bridge can be found in the above figure 2(c).
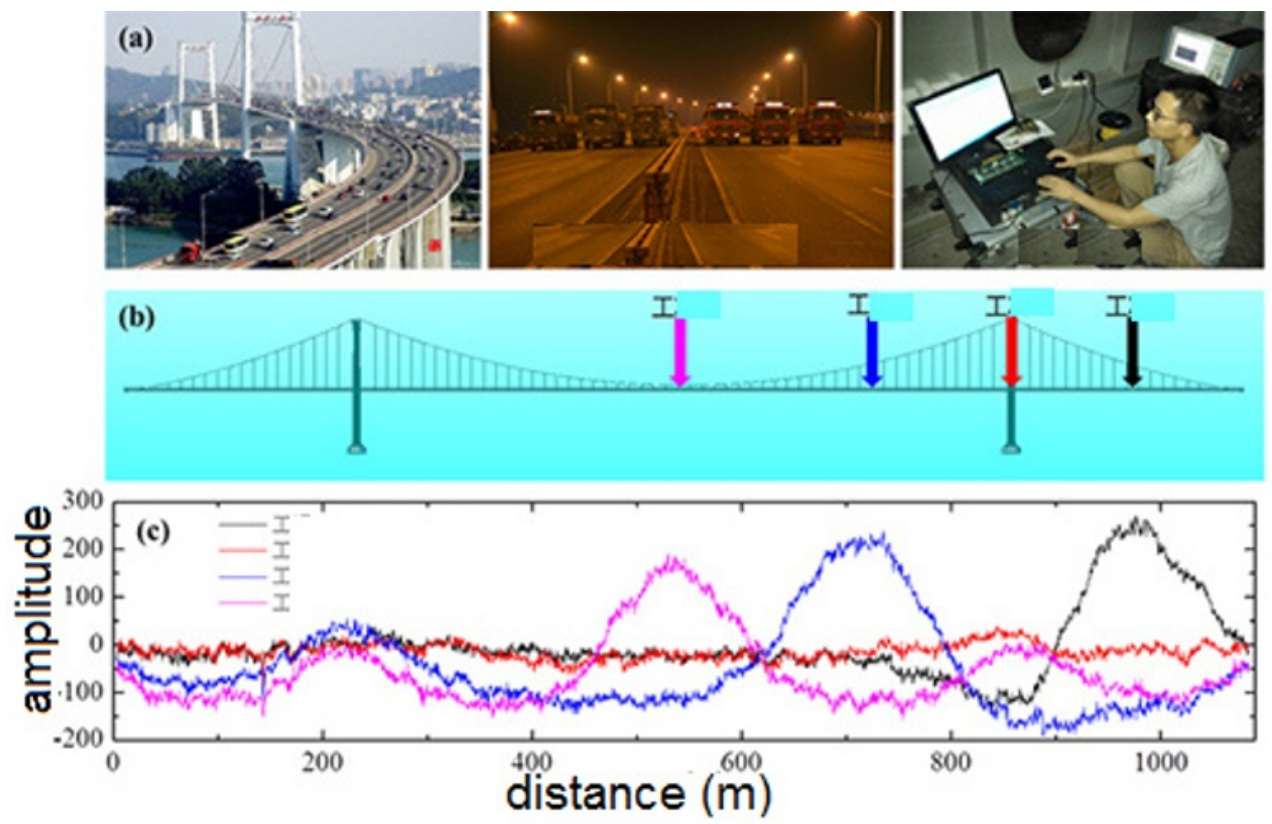

Fig. 2. Xia Men Haicang Bridge Loading Monitoring 


\section{Conclusion}

1.Distributed optical fiber sensing technology has the advantages of high precision, many measuring points, strong reliability, anti-interference, corrosion resistance, wide measurement range and long-distance monitoring, etc. It has great development potential and can be widely applied in various geotechnical engineering monitoring fields.

2.The monitoring data confirms the correctness of the design decisions both for the predicted efforts in the elements of the bridge structure, and for the installation of the cablestayed beam system, which is able to withstand hurricane winds in the area of bridge crossings.

The work is funded by National Key R\&D Program of China (2016YFE0202400) to create a monitoring system for unique buildings and structures in special conditions.

\section{Reference}

1. 1 Wang Xing, Shi Bin et al. Chinese Journal of Geotechnical Investigation \& Surveying.2015, $12: 13 \sim 17$.

2. 2 Wei Guangqing, Shi Bin et al. Chinese Journal of Engineering Geology, 2008, 16 ( 6 ) : $826 \sim 832$.

3. 3 Liu J, Shi B, Zhang D, et alRock and Soil Mechanics, 2006, 27(7): 1224-1228.

4. 4 Sui Haibo, SHI bin et al. Chinese Journal of Rock Mechanics and Engineering, 2008,Vol.27,Supp.2:3725-3729.

5. Kudryavtsev S.A., Arshinskaya L.A., Valtseva T.U., Berestyanyy U.B., Zhusupbekov A.Z. Proceedings of the International Workshop on Scrap Tire Derived GeomaterialsOpportunities and Challenges, IWTDGM 2007. Yokosuka, 2008. P. 171-178.

6. Kudryavtsev S.A., Berestyanyy.U.B, Goncharova E.D, Proc. of the International Offshore and Polar Engineering Conference. "Proceedings of the 23rd International Offshore and Polar Engineering Conference, ISOPE 2013" 2013. P. 562-566.

7. Kudryavtsev S.A., Berestyanyy Y.B., Valtseva T.Y., Goncharova E.D., Mikhailin R.G. Proc. of the Int. Conf. on Cold Regions Engineering. "Cold Regions Engineering 2009: Cold Regions Impacts on Research, Design, and Construction" 2009. P. 546.

8. Paramonov V., Sakharov I., Kudriavtcev S. MATEC Web of Conferences 2016. 05007.

9. Kudriavtcev S., Paramonov V., Sakharov I. MATEC Web of Conferences 2016. P. 05002.

10. Kudryavtsev S., Kazharsky A.V., Valtseva T., Kotenko Z., Goncharova E.. Procedia engineering. 2016. 165. 1080-1086. 